\title{
Gaucher Disease for the Haematologist
}

\author{
An Expert Interview with Maria Domenica Cappellini \\ Centro Malattie Rare, Ca Granda Foundation IRCCS, Policlinico Hospital, Milan, Italy; Department of Clinical Sciences and Community Health, \\ University of Milan, Milan, Italy
}

DOI: https://doi.org/10.17925/EOH.2019.15.1.14

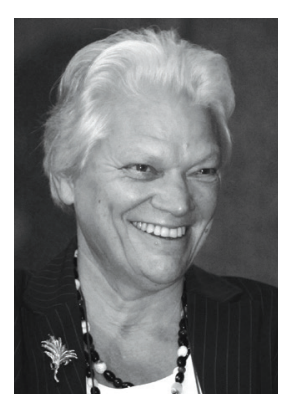

\begin{abstract}
Maria Domenica Cappellini
Maria Domenica Cappellini is Professor of Internal Medicine at the University of Milan and Chief of the Rare Diseases Centre at the Fondazione IRCCS Policlinico Hospital, Milan, Italy. She qualified as a doctor of medicine (MD) in 1974 at the University of Milan, Italy, and has been active in the fields of thalassaemia, haemoglobinopathies and the haematological biosynthetic pathway for over 40 years. She is also a member of the European Gaucher Registry. Prof Cappellini has been an active researcher in the field of rare diseases, including thalassaemia, haemoglobinopathies, porphyrias and lysosomal diseases (mainly Gaucher and Fabry diseases) for over 20 years. She has focused on the phenotypic expression of thalassaemia intermedia and identified the mechanisms underlying the thrombotic risks associated with the disease. She is involved in clinical trials of new iron chelators and has focused recent studies on iron dysregulation in Gaucher disease. Prof Cappellini has published a large number of peer-reviewed original articles in these areas and is a regular contributor and invited speaker at national and international meetings. She contributed to the characterisation of the molecular defects of thalassaemia globin genes in Italy; of note, she defined the genotypes of Italian patients with thalassaemia intermedia that became nationally important for prenatal diagnosis. In addition, she developed an erythroid liquid culture system from peripheral erythroid progenitors in order to study molecules with the potential capability of reversing haemoglobin switching, or inducing foetal haemoglobin production in view of therapeutic application.
\end{abstract}

She is involved in clinical trials for new iron chelators and new molecules for correcting ineffective erythropoiesis. Prof Cappellini is a member of a number of societies, including the European Hematology Association (EHA), the American Society of Hematology (ASH), the Italian Society of Hematology (SIE), the Italian Society of Internal Medicine (SIMI) and the International Biolron Society (IBIS). She is scientific advisor to the Thalassaemia International Federation (TIF) and contributes to establish guidelines for the clinical management of thalassaemia. Prof Cappellini is a past president of the European Federation of Internal Medicine (EFIM). She is also a member of the European School of Haematology Strategy Board (Paris, France) and a member of EHA's Fellowships \& Grants Committee for EHA 2018. She is an honorary fellow of the Royal College of Physicians and American College of Physicians. She received a Sultan Bin Khalifa International Thalassemia Award - Grand International Award, in Abu Dhabi, United Arab Emirates in November 2015 and the 'Precious Rubies' award from the Thalassaemia International Federation in Nicosia, Cyprus in May 2015.

\section{Keywords}

Gaucher disease, diagnosis, dried blood, spot assay, rare haematological disorders

Disclosure: Maria Domenica Cappellini has received honoraria for advisory boards from Novartis Pharmaceuticals, Sanofi Genzyme and Celgene.

Acknowledgements: Medical writing support was provided by Katrina Mountfort of Touch Medical Media and was supported by Touch Medical Media. Review Process: This is an expert interview and, as such, has not undergone the journal's standard peer review process.

Compliance with Ethics: This article is an opinion piece and does not report on new clinical data, or any studies with human or animal subjects performed by any of the authors.

Authorship: The named author meets the International Committee of Medical Journal Editors (ICMJE) criteria for authorship of this manuscript, takes responsibility for the integrity of the work as a whole, and has given final approval for the version to be published.

Received: 17 October 2018

Accepted: 20 February 2019

Citation: European Oncology \& Haematology. 2019; 15(1):14-5

Corresponding Author: Maria-Domenica Cappellini, Fondazione Ca Granda Ospedale Maggiore Policlinico IRCCS, University of Milan, Via F. Sforza 35, 20122 Milano, Italy. E: maria.cappellini@unimi.it

Support: No funding was received in the publication of this article. aucher disease is the most common of the lysosomal storage disorders." Although individually rare, these disorders as a group are relatively common, with an incidence of about 1 in 8,000 live births, ${ }^{2}$ and therefore represent an important health problem. Gaucher disease is characterised by an enzyme deficiency causing the deposition of glucocerebroside in cells of the macrophage-monocyte system. Since its clinical presentation can range from being asymptomatic to life-threatening symptoms, it can be difficult to diagnose. In an expert interview, Maria Domenica Cappellini discusses the challenges of diagnosing Gaucher disease and the characteristic features that physicians should look for.

\section{Q. What is Gaucher disease?}

Gaucher disease is the most common inherited lysosomal disorder due to a deficiency of acid beta-glucosidase (glucocerebrosidase). This leads to glucosylceramide accumulation in lysosomes of macrophages. The glycolipid-laden cells, named Gaucher cells, infiltrate organs to cause multisystem disease. So far, the disease is still classified into three subgroups: type 1 : non neuronopathic (94\%); type 2: acute neuronopathic (1\%; death in infancy); and type 3: chronic neuronopathic (5\%; death in childhood/early adulthood).

\section{Q. Why is early diagnosis so important and why is it often delayed in this condition?}

Early diagnosis is important in order to define the disease manifestations and to start treatment as soon as possible in order to prevent disease progression. The delay in diagnosis is mainly due to the fact that Gaucher disease is rare and many physicians do not include Gaucher disease in differential diagnosis because they are unaware of the condition and they assume that Gaucher disease is so rare they are unlikely to encounter a case in their clinical practice. Gaucher disease is often only considered when other more likely possibilities have been excluded. The incidence is 1 in 40,000-60,000, and 1 in 850 in individuals of Ashkenazi Jewish background. ${ }^{3}$ Moreover, Gaucher disease is a phenotypically heterogeneous disease. There is an enormous variation in the age of onset, rate of progression, organs affected, disease severity across individuals, severity of disease across organs in one individual and presenting symptoms, even in individuals 
of the same genotype. Evidence indicates that individuals with Gaucher disease experience delays of up to 10 years before diagnosis. ${ }^{4}$ The delay in diagnosis may be due to unimpeded disease progression, increased risk of complications, avascular necrosis, uncontrolled bleeding (especially after surgery), pulmonary hypertension, growth retardation, fatal septicaemia, pathological fractures, bone collapse, hepatosplenomegaly, unnecessary or inappropriate investigations, inappropriate treatment and delayed access to appropriate therapy.

\section{Q. What characteristic disease features should physicians be looking for?}

The most common characteristics at any time in a patient with Gaucher disease type 1 are splenomegaly and thrombocytopenia. These may be associated to anaemia, mild leucopoenia, bone pain, monoclonal gammopathy and hepatomegaly. Various other less-specific abnormalities may be supportive for Gaucher disease diagnosis such as gall stones or cholelithiasis, low cholesterol, hyperferritinemia, pregnancy-associated thrombocytopenia and post-partum haemorrhage. For any physician, suspicion of Gaucher disease is appropriate when family history, patient history or investigations include bruising, bone pain, fatigue, abdominal pain, splenomegaly and laboratory evidence of thrombocytopenia and/ or anaemia; or unexplained stable hyperferritinemia with normal transferrin saturation, increased inflammatory markers, failure to thrive and/or delayed growth in childhood, gall stones/cholelithiasis or low cholesterol, splenic nodules, fractures, premature osteoporosis or avascular necrosis; or pregnancy-associated thrombocytopenia and/or post-partum haemorrhage.

\section{Q. What diagnostic tests are available?}

The laboratory diagnostic tests available include macrophage-specific biochemical markers, the most commonly used of which include chitotriosidase and LysoGB1; dried blood spot; specific enzyme dosage (glucocerebrosidase); and molecular analysis. Beside the laboratory tests, an accurate family history and clinical evaluation is essential.

\section{Q. What are the treatment options for Gaucher disease?}

Today, the treatment options for Gaucher disease are enzyme replacement therapy and substrate reduction therapy. Any adjunctive medication or intervention must be considered for treating the comorbidities.

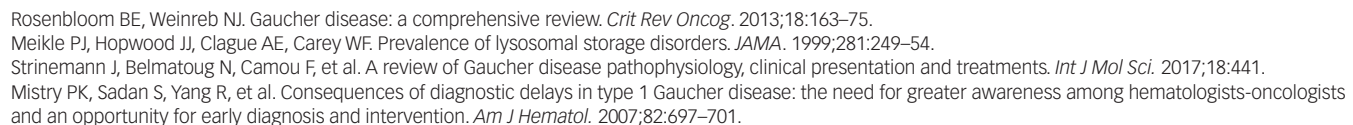

